УДК 621.039 .5

\title{
ПЕРСПЕКТИВНЫЕ ИССЛЕДОВАНИЯ ИННОВАЦИОННЫХ ТЕХНОЛОГИЙ ЯДЕРНЫХ ЭНЕРГЕТИЧЕСКИХ УСТАНОВОК
}

\author{
Авраменко А.А., член-корреспондент НАН Украины, Ковецкая М.М., к.т.н., \\ Кравчук А.В., Ковецкая Ю.Ю.
}

Институт технической теплофизики НАН Украинь,, ул. Желябова 2а, Киев 03680, Украина

\begin{abstract}
Представлено огляд перспективних технологій ядерних енергетичних установок для розв'язання задач підвищення безпеки i економічної ефективності. Показано роль теплофізичних досліджень для удосконалення легководних ядерних реакторів.
\end{abstract}

Представлен обзор перспективных технологий ядерных энергетических установок для решения задач повышения безопасности и экономической эффективности. Показана роль теплофизических исследований для совершенствования легководных ядерных реакторов.
A review of perspective nuclear power technologies is presented for solving the problems of improving the safety and economic efficiency. A role of thermal physical investigation for improving light-water reactors is shown.

Библ. 54, табл. 5.

Ключевые слова: ядерные реакторы нового поколения, гелиевый теплоноситель, вода сверхкритического давления, теплофизические исследования.

ВВЭР - водо-водяной энергетический реактор;

ВБЭР - водяной блочный энергетический реактор; ВТГР - высокотемпературный газоохлаждаемый

\section{введение}

Состояние и перспективы развития технологий ядерных энергетических установок в странах мира постоянно анализируются и отражаются в многочисленных публикациях, например в [1-6]. Создаются международные проекты по разработке перспективных ядерных энергетических систем, включая как ядерные реакторы, так и топливные циклы. Так международная программа GIF IY (Generation IY International Forum) функционирует с 2001 г. и направлена на разработку ядерных энергетических систем четвертого поколения с высокими эксплуатационными характеристиками, повышенной экономичностью и безопасностью.

МАГАТЭ осуществило международный проект INPRO (International Project on Innovative Nuclear Reactors and Fuel Cycles) по разработке ядерных реакторов и топливных циклов, который направлен на создание критериев выбора различных инновационных технологий исходя из реактор;

СКД - сверхкритическое давление;

твэл - тепловыделяющий элемент.

требований пользователей.

Каждая страна выбирает свое направление развития атомной энергетики на основе прогнозов экономического развития и исходя из принципов безопасности, конкурентоспособности, наличия или доступности топливных ресурсов, способа утилизации ядерных отходов, нераспространения ядерного оружия.

Наиболее важными задачами ядерной энергетики XXI века по мнению авторов работы [7] являются:

- создание замкнутого топливного цикла с расширенным воспроизводством и повторным использованием плутония и ${ }^{233} \mathrm{U}$ :

- безопасное обращение с радиоактивными отходами, включая создание хранилищ отработанного ядерного топлива;

- создание эффективного реактора-размножителя, ядерных энергетических установок для теплоснабжения и технологических производств.

Украина окончательно не определилась с 
выбором своего ядерного топливного цикла [8]. Согласно «Энергетической стратегии Украины до 2030 г.» основное внимание будет уделяться продлению срока эксплуатации действующих энергоблоков, исчерпавших свой проектный ресурс. Предполагается также строительство новых энергоблоков с легководными реакторами поколения $\amalg^{+}$. В работе [8] определены задачи, стоящие перед Украиной по созданию ядерного топливного цикла и в частности отмечается, что для эффективного использования продуктов отработанного ядерного топлива, в структуре ядерной энергетики должны быть реакторы как на тепловых, так и на быстрых нейтронах.

\section{Программа GIF IY}

В 70-е годы прошлого столетия в ядерной технологии было введено понятие «внутренне присущей безопасности», предусматривающее максимальное использование присущих ядерному топливу, теплоносителю, радиоактивным отходам и другим компонентам фундаментальных физических и химических свойств и закономерностей.

Такой подход позволяет достичь высокой безопасности ядерного объекта не усложнением, а упрощением конструкций.

В рамках программы GIF IY были выбраны шесть инновационных ядерных реакторов, которые могут быть введены в эксплуатацию до 2030 г. [9-12]:

- быстрый реактор с газовым (гелиевым) теплоносителем GFR (Gas Fast Reactor) с замкнутым топливным циклом;

- высокотемпературный реактор с гелиевым теплоносителем и графитовым замедлителем VHTR (Very High Temperature Reactor);

- реактор с водой сверхкритических параметров SCWR (Supercritical Water Reactor);

- быстрый реактор с натриевым теплоносителем SFR (Sodium Fast Reactor), замкнутым топливным циклом, эффективным сжиганием актиноидов, воспроизводством топлива на основе урана;

- быстрый реактор со свинцовым/свинцово-висмутовым теплоносителем LFR (Lead Fast Reactor), замкнутым топливным циклом, сжиганием актиноидов, эффективным воспроизвод- ством ядерного топлива.

- peактор на тепловых нейтронах MSR (Molten Salt Reactor) с циркуляцией топлива на основе расплавленных солей, с полной переработкой актиноидов в топливном цикле.

При разработке этих проектов основное внимание уделяется следующим аспектам:

- экономической конкурентоспособности, включая стоимость топливного цикла;

- ужесточению мер по ограничению распространения ядерных оружейных материалов;

- повышению эффективности технологий переработки ядерных отходов;

- повышению степени использования ресурсов ядерного топлива;

- совершенствованию эффективности ядерных технологий.

Среди этих технологий выделяются две технологии производства электроэнергии с КПД $45 . .47 \%$ использующие высокотемпературные реакторы с гелиевым теплоносителем и реакторы, охлаждаемые водой сверхкритического давления.

\section{Высокотемпературные газовые реакторы} (ВТГР)

Разработка ВТГР осуществляется по следующим направлениям: энергоблоки с паровым циклом (температура теплоносителя на выходе $\left.750{ }^{\circ} \mathrm{C}\right)$; с прямым газотурбинным циклом (температура на выходе $850{ }^{\circ} \mathrm{C}$ ); для получения технологического тепла (температура на выходе $950{ }^{\circ} \mathrm{C}$ и более).

Высокий уровень безопасности ВТГР достигается за счет сочетания свойств внутренне присущей безопасности и конструктивных решений:

- гелиевый теплоноситель не влияет на реактивность;

- графит, используемый в качестве замедлителя и конструкционного материала активной зоны, в результате высокой теплоемкости аккумулирует теплоту, выделяемую в топливе;

- топливо на основе микрочастиц с термостойким покрытием надежно удерживает радиоактивные продукты деления;

- отрицательный коэффициент реактивности по температуре способствует уменьшению мощ- 
ности реактора при росте температуры теплоносителя.

Проекты высокотемпературных гелиевых реакторов электрической мощностью 1000 МВт для промышленных АЭС разрабатываются в США (HTGR-1160), Германии (THTR-1000), Великобритании (HTGR). Исследования показали, что уровень самозащищенности реактора ВТГР тем выше, чем меньше размер реактора, плотность энерговыделения и температура топлива. В этом случае появляется возможность организовать отвод остаточного тепловыделения пассивными средствами (естественная конвекция, теплопроводность, излучение). В рамках программы GIF IY разрабатываются проекты быстрых реакторов для производства электроэнергии, решения задач минимизации высокоактивных отходов, производства водорода. В табл. 1 представлены технические параметры реакторов с гелиевым теплоносителем для высокотемпературного термохимического производства, а также выработки электроэнергии, разрабатываемые в США [9]:

Таблица 1

\begin{tabular}{|l|c|c|}
\hline Параметры & GFR & VHTR \\
\hline Тепловая мощность, МВт & 600 & 600 \\
\hline Температура теплоносителя, вход/выход, ${ }^{\circ} \mathrm{C}$ & $790 / 850$ & $640 / 1000$ \\
\hline Давление, МПа & 9 & 7 \\
\hline Массовый расход, кг/с & 320 & 320 \\
\hline Спектр нейтронов & быстрый & тепловой \\
\hline КПД & 48 & $>50$ \\
\hline
\end{tabular}

Реакторы GFR на быстрых нейтронах с гелиевым теплоносителем предназначены для эффективного преобразования делящегося урана и сжигания актиноидов, организации пристанционного замкнутого топливного цикла [10]. РеакTop VHTR на тепловых нейтронах с гелиевым теплоносителем и уран-плутониевым топливным циклом предназначен для выработки электроэнергии и технологического тепла. В активной зоне реактора могут использоваться как призматические тепловыделяющие сборки, так и шаровая засыпка.

Основными проблемами высокотемператур- ных реакторов для получения электроэнергии и технологического тепла зарубежные специалисты считают выбор типа твэлов, создание конструкционных материалов для теплообменных и теплотранспортных систем, работающих в условиях очень высоких температур, повышение надежности и безопасности эксплуатации. В Южноафриканской республике разрабатывается модульный реактор PBMR (Particle Bed Modular Reactor) на тепловых нейтронах с гелиевым теплоносителем и насыпной активной зоной. Его основные технические характеристики приведены в табл. 2 [13].

Таблица 2

\begin{tabular}{|l|c|c|}
\hline & PBMR & GT-MHR \\
\hline Тепловая мощность, МВт & 265 & 600 \\
\hline Электрическая мощность, МВт & 117 & 285 \\
\hline КПД, \% & 44,2 & 47,5 \\
\hline Температура на входе/выходе, ${ }^{\circ} \mathrm{C}$ & $536 / 900$ & $491 / 850$ \\
\hline Давление, МПа & 7 & 7 \\
\hline Расход гелия, кг $/$ с $^{\text {. }}$ & 140 & 320 \\
\hline Среднее выгорание топлива, ГВт.су/т & 80 & \\
\hline
\end{tabular}


Технологическая схема энергоблока - одноконтурная с прямым газотурбинным циклом Брайтона. Реактор и все системы первого контура размещены в корпусе под давлением, а газовая турбина, электрогенератор, компрессоры и теплообменники - в отдельном блоке. При использовании сферических топливных элементов движение топлива происходит в реакторе под нагрузкой. Таким образом устраняется необходимость в остановках реактора для перегрузок топлива, которые снижают коэффициент использования установленной мощности. Среднее обогащение топлива составляет 5..6 \%, а выгорание достигает 80 ГВт.сут/т.

В США осуществляется более крупный проект газотурбинного модульного гелиевого реактора GT-MHR (Gas Turbine - Modular Helium Reactor) электрической мощностью 285 МВт. Технологическая схема энергоблока - одноконтурная с газотурбинным приводом электрогенератора. Технические характеристики GT-MHR приведены в табл. 2.

Существенное преимущество энергоблока высокий уровень ядерной безопасности. Низкая плотность энерговыделений в активной зоне и конструктивное блочно-модульное исполнение обеспечивают пассивное, без каких либо систем аварийного расхолаживания, рассеивание остаточных тепловыделений в окружающую среду без повреждения твэлов.

B активной зоне GT-MHR может быть использовано топливо на основе урана с применением в качестве делящегося материала оружейного плутония. По мнению американских специалистов выгорание плутония будет достигать 95 \%. Это обстоятельство является весьма привлекательным, так как уменьшает опасность распространения ядерного оружия. Проект разрабатывают компания General Atomics (США) в сотрудничестве с Российским Минатомом при поддержке компаний Франции и Японии [14].

Учитывая перспективность высокотемпературных газовых реакторов для производства электроэнергии и высокотехнологического тепла, нами были проведены исследования теплофизических процессов в элементах активной зоны реакторов для оценки их безопасности. Исследованы закономерности и особенности теплообмена при течении гелия в активной зоне стержневого и насыпного типа. Была усовершенствована трехмерная математическая модель турбулентного тепломассообмена на основе ренормализационно-группового похода для исследования нестационарных процессов и учета эффектов микропористой среды $[15,16]$.

При исследовании гидродинамических и теплообменных процессов в модели активной зоны реактора насыпного типа с шаровыми тепловыделяющими элементами установлена зависимость температуры оболочки твэлов от проницаемости засыпки; получены распределения профилей скорости и температуры в активной зоне в зависимости от проницаемости пористой среды; определены критерии гидродинамической неустойчивости. Полученные результаты могут быть использованы для оценки безопасности высокотемпературных гелиевых реакторов с шаровыми тепловыделяющими элементами.

Исследованы также закономерности гидродинамики и теплообмена в активной зоне высокотемпературных гелиевых реакторов с гексагональными тепловыделяющими сборками стержневого типа. Расчеты проведены для модельной семистержневой тепловыделяющей сборки треугольной упаковки в стационарных и нестационарных режимах. Получены распределения скоростей и температур по ячейкам пучка обогреваемых стержней, определено влияние необогреваемой стенки и межканальных перетечек на параметры теплоносителя. Исследована азимутальная неравномерность температуры обогреваемых стержней и ее зависимость от входных параметров. Минимальные значения коэффициентов теплоотдачи наблюдаются в угловом зазоре между тепловыделяющим стержнем и стенкой [17].

Исследованы нестационарные режимы с уменьшением расхода теплоносителя и скачками тепловой мощности. Показано влияние изменения расхода теплоносителя в тепловыделяющей сборке с учетом поверхностного и объемного тепловыделения на распределение температуры. Определено время достижения температуры стенки обогреваемого стержня предельно допустимого значения [18]. Проанализированы опасные режимы, возникающие при 
всплесках мощности. Знание локальных характеристик течения и теплообмена в активной зоне реактора позволяет оценить его надежность и безопасность.

\section{Реакторы, охлаждаемые водой сверхкритического давления}

Основная задача использования легководных реакторов со сверхкритическим давлением SCWR состоит в том, чтобы повысить экономичность существующих технологий с реакторами PWR (Pressurized Water Reactor). Эта концепция реакторов рассматривается специалистами России, Японии, США, Франции, Канады, Китая и других стран в рамках международных программ по разработке реакторов четвертого поколения. Строительство АЭС с реакторами SCWR позволит повысить КПД установок до $44 . .48 \%$, снизить металлоемкость оборудования по сравнению с существующими АЭС с ВВЭР или PWR [19-21]. Рассматриваются концепции реакторов корпусного и канального типа, с тепловым и быстрым спектром нейтронов. Ядерные энергоблоки с реактором SCWR характеризуются следующими особенностями [20]:

- низким расходом теплоносителя и высоким его подогревом в активной зоне, что позволяет снизить мощность, потребляемую циркуляционными насосами;

- высокой энтальпией теплоносителя, что обеспечивает высокий КПД установки;

- однофазностью теплоносителя, следовательно, отсутствием режимов с кризисом теплообмена;

- реализацией прямоточного цикла, что приводит к компактности реакторной системы;

- возможностью использования технологий и оборудования энергоблоков СКД с органическим топливом.

Возможны варианты компоновки активной зоны с тепловым спектром нейтронов для эксплуатации в открытом топливном цикле с топливом $\mathrm{UO}_{2}$ и быстрым спектром нейтронов для работы в замкнутом топливном цикле с МOX-топливом и коэффициентом воспроизводства КВ $\approx 1$ или выше, до 1,2 [22].

На основании предварительных проектных разработок легководных реакторов со сверхкритическим давлением, выполненных в США, Франции и России в табл. 3 представлены их основные характеристики.

Таблица 3

\begin{tabular}{|l|c|c|c|}
\hline Параметр & $\begin{array}{c}\text { HPLWR } \\
\text { (Франция) }\end{array}$ & $\begin{array}{c}\text { SCWR } \\
\text { (США) }\end{array}$ & $\begin{array}{c}\text { ВВЭР-СКД } \\
\text { (Россия) }\end{array}$ \\
\hline Электрическая мощность, МВт & 1000 & 1600 & 1700 \\
\hline Температура теплоносителя, вход/выход, ${ }^{\circ} \mathrm{C}$ & $280 / 500$ & $280 / 500$ & $280 / 530$ \\
\hline Давление теплоносителя, МПа & 25 & 25 & 25 \\
\hline Диаметр и толщина оболочки твэла, мм & $8 \mathrm{x} 0,4$ & $10,2 \mathrm{x} 0,63$ & $10,7 \mathrm{x} 0,55$ \\
\hline Шаг между твэлами, мм & 9,5 & 11,2 & 12,0 \\
\hline Обогащение топлива UО,$\%$ & $\leq 5$ & $\leq 5$ & $\leq 5$ \\
\hline Среднее выгорание топлива, МВт.сут/кгU & 45 & 45 & Около 40 \\
\hline Максимальная температура оболочки твэла, ${ }^{\circ} \mathrm{C}$ & \multicolumn{3}{|c|}{ Около 670} \\
\hline
\end{tabular}

Особенности охлаждения активной зоны водой сверхкритических параметров учитываются при формировании спектра нейтронов, анализе безопасности и оценке устойчивости работы реактора. В табл. 4 представлены основные характеристики разрабатываемых в России одноконтурной и двухконтурной реакторных установок
ВВЭР-СКД с естественной (ЕЦ) и принудительной (ПЦ) циркуляцией теплоносителя [23].

В Канаде ведутся разработки усовершенствованных тяжеловодных канальных реакторов CANDU-X (Canada Deuterium Uranium -X) c водой сверхкритических параметров в качестве теплоносителя электрической мощностью от 
375 до 1200 МВт (табл. 4) [9]. Предложена новая конструкция топливного канала, в котором замедлитель (тяжелая вода) отделяется от теплоносителя высокого давления циркониевой трубой с внутренним теплоизолирующим слоем.

Проект реактора SCWR на быстрых нейтронах электрической мощностью 700 МВт на суперкритические параметры разработан в Японии [20]. Активная зона рассчитана на высокую плотность энерговыделения при максимальной линейной тепловой нагрузке, не превышающей
39 кВт/м. При расходе теплоносителя 850 кг/с эквивалентный диаметр активной зоны составляет 2,1 м, что значительно меньше, чем у реакторов PWR такой же мощности. Активная зона реактора содержит запальные и бланкетные сборки. Запальные сборки генерируют быстрые нейтроны и служат источником тепловой энергии, бланкетные сборки используются для обеспечения отрицательного пустотного коэффициента реактивности. В табл. 5 представлены расчетные параметры активной зоны реактора SCWR [20].

Таблица 4

\begin{tabular}{|c|c|c|c|}
\hline Параметр & ВВЭР-СКД & ВВЭР-СКД & CANDU-X \\
\hline Тип установки & Одноконтурная & Двухконтурная & \\
\hline Спектр нейтронов & Тепловой, быстрый & Тепловой & Тепловой \\
\hline Тепловая мощность , МВт & 3700 & $\begin{array}{l}1250 \text { (ЕЦ) } \\
2500(П Ц)\end{array}$ & 2500 \\
\hline Электрическая мощность, МВт & 1700 & $\begin{array}{l}500 \text { (ЕЦ) и } \\
1000 \text { (ПЦ) }\end{array}$ & 1200 \\
\hline КПД,\% & 45 & 43 & 48 \\
\hline Давление, МПа & 24,5 & 24,5 & 25 \\
\hline Температура на входе/ выходе, ${ }^{\circ} \mathrm{C}$ & $290 / 540$ & $390 / 540$ & $350 / 625$ \\
\hline Топливо & $\mathrm{UO}_{2}, \mathrm{MOX}$, кермет $\left.{ }^{*}\right)$ & $\mathrm{UO}_{2}, \mathrm{MOX}$ & $\mathrm{UO}_{2} / \mathrm{Th}$ \\
\hline Обогащение, \% & $\begin{array}{l}\text { до } 5 \text { (тепловой), } \\
\text { до } 20 \text { (быстрый) }\end{array}$ & & 4 \\
\hline Диаметр твэла, мм & $9 \ldots 11$ & $9 \ldots 11$ & 7 \\
\hline
\end{tabular}

*) - керметное топливо с дополнительным барьером для одноконтурной установки

Таблица 5

\begin{tabular}{|l|c|}
\hline Параметры & \\
\hline Тепловая мощность, МВт & 1650 \\
\hline Электрическая мощность, МВт & 720 \\
\hline Высота активной зоны, см & 300 \\
\hline Эквивалентный диаметр активной зоны, см & 210 \\
\hline Обогащение по делящемуся Ри, масс.\% & 24,87 \\
\hline Температура теплоносителя на выходе активной зоны, ${ }^{\circ} \mathrm{C}$ & 503,7 \\
\hline Максимальная температуры поверхности оболочки твэлов, ${ }^{\circ} \mathrm{C}$ & 639,8 \\
\hline Средняя линейная тепловая нагрузка, кВт/м & 17,3 \\
\hline Расход теплоносителя, кг/с & 850,0 \\
\hline Среднее выгорание топлива, МВт.сут/кг ТМ & 69,3 \\
\hline
\end{tabular}


При освоении технологии леководных реакторов со сверхкритическими параметрами теплоносителя необходимо полное понимание теплогидравлических процессов в теплоносителе и решение проблемы создания новых конструкционных материалов активной зоны. В теплофизическом плане одной из важных является проблема определения коэффициента теплоотдачи в активной зоне реактора и безопасной области тепловых нагрузок, чтобы исключить возможность перехода в режим ухудшенного теплообмена. В режиме ухудшенного теплообмена наблюдается резкое снижение коэффициента теплоотдачи с образованием зон перегрева, что может привести к разрушению оболочки тепловыделяющих элементов. Поэтому исследование природы явления ухудшенного теплообмена при переходе от докритических к сверхкритическим параметрам теплоносителя и разработка надежных методов его прогноза важны для обеспечения безопасности реакторной установки.

Результаты экспериментальных и теоретических исследований природы ухудшенного теплообмена при течении воды СКД в каналах свидетельствует о том, что это явление имеет гидродинамическую природу и связано с существенным изменением структуры как осредненного, так и пульсационного течения [25]. Резкое увеличение теплоемкости и уменьшение плотности среды при приближении к критической температуре вызывает термическое ускорение потока, что приводит к уменьшению турбулентности вблизи стенки. При подъемном течении теплоносителя в трубах профиль скорости приобретает седлообразную форму с максимумом вблизи стенки, где образуется запирающий для переноса теплоты слой. Это приводит к ухудшению теплообмена и резкому повышению температуры стенки. Актуальным является вопрос насколько опасны такие режимы для тепловыделяющих сборок и при каких геометрических и режимных параметрах они возникают.

Экспериментальных исследований теплообмена и гидродинамики в тепловыделяющих сборках с водой сверхкритических параметров очень мало. Для обоснования безопасности таких энергоблоков проводят расчеты возможных аварийных режимов на основе трехмерных ма- тематических моделей теплообмена и гидродинамики в активной зоне реакторов и одномерных моделей с поканальным (ячейковым) теплогидравлическим расчетом [24].

В работах $[15,18,26]$ представлены результаты численного моделирования теплообмена и гидродинамики при течении воды сверхкритического давления в вертикальной семистержневой тепловыделяющей сборке с параметрами активной зоны ВВЭР-СКД. Численное моделирование течения теплоносителя в сборке выполнено на основе RNG k- $\varepsilon$ модели турбулентности, включающей систему уравнений сохранения массы, количества движения, энергии теплоносителя, а также уравнения для кинетической энергии турбулентности и скорости диссипации энергии.

Определены локальные характеристики потока в ячейках пучка обогреваемых стержней, изменение температуры стенки и коэффициента теплоотдачи по длине и сечению тепловыделяющей сборки, влияние необогреваемой стенки на характеристики потока, условия возникновения режима ухудшенного теплообмена. Распределение коэффициента теплоотдачи по длине тепловыделяющей сборки в теплонапряженной точке носит немонотонный характер. Максимум коэффициента теплоотдачи наблюдается в области достижения температуры теплоносителя критического значения. После достижения максимума начинается резкое падение коэффициента теплоотдачи в соответствии с уменьшением теплоемкости и термическим ускорением потока вблизи обогреваемой стенки. В этой области происходит ламинаризация потока, образуется запирающий для переноса теплоты слой и возникает режим ухудшенного теплообмена, характеризующийся ростом температуры стенки. В сечении, где температура теплоносителя достигает критического значения, наблюдается существенная неравномерность коэффициента теплоотдачи по окружности тепловыделяющего элемента.

Исследованы нестационарные режимы, характеризующиеся уменьшением расхода теплоносителя и резким увеличением тепловой мощности. Определены границы области ухудшенного теплообмена и их перемещение в нестационарных режимах. Определены условия, при которых температура стенки тепловыделяющего 
элемента достигает предельно допустимого значения.

\section{Ядерные реакторы малой и средней мощиности (SMR)}

МАГАТЭ отмечает возобновление интереса к реакторам малой и средней мощности SMR (Small and Medium Reactor) для производства электроэнергии, теплоснабжения, опреснения морской воды, производства водорода и других технологических процессов [27-31]. По определению МАГАТЭ к реакторам малой мощности относятся реакторы электрической мощностью до 300 МВт, к реакторам средней мощности реакторы электрической мощностью до 700 МВт. Предпосылками для развития реакторов малой и средней мощности являются следующие факторы:

- повышение надежности и безопасности за счет внутренне присущей безопасности и упрощения конструкции;

- обеспечение масштабного эффекта за счет роста числа модулей, возможность наращивания мощности электростанций по мере потребностей добавлением новых модульных единиц;

- замена устаревших энергоблоков на ископаемом топливе;

- экономическая доступность за счет заводского производства модулей и сокращения сроков строительства энергоблоков;

- привлекательность для энергоснабжения отдаленных районов.

Особый интерес инвесторов вызывают малые реакторы модульной конструкции с большой длительностью кампании [30]. Малые габариты и гарантированная безопасность позволяют расположение энергоблоков в границах населенных пунктов на площадках с наземным и подземным размещением. Такие ядерные энергетические установки обеспечивают затрудненный несанкционированный доступ к топливу во время его использования на площадке и транспортирования.

В настоящее время разрабатываются более трех десятков проектов модульных реакторов в разных странах. Большинство из них - с легководным теплоносителем. Из наиболее разработанных отмечаются проекты реактора SMART
(System-integrated Modular Advanced Reactor) (Южная Корея), электрической мощностью 100 МВт, для производства электроэнергии, теплоснабжения и опреснения морской воды; ВБЭР-300 (Россия) электрической мощностью $150,230,300$ МВт (в зависимости от числа петель), для производства электроэнергии и теплоснабжения; Westinghouse SMR (США), электрической мощностью $225 \mathrm{MBT,} \mathrm{для} \mathrm{замены}$ стареющих генерирующих мощностей, работающих на ископаемом топливе [32]. Ядерная часть энергоблока с реактором Westinghouse SMR и бассейн с отработанным топливом располагаются под землей для защиты от внешних угроз. Заводское изготовление и сборка основного оборудования, сжатые графики строительства обеспечивают минимальные сроки между инвестициями и началом поступления денег от эксплуатации электростанции, что обеспечивает инвестиционную привлекательность проекта.

Проекты энергоблоков с реакторами малой и средней мощности разрабатываются также в Аргентине, Китае, Индии, Пакистане, Японии. Для развитых стран SMR дают возможность сооружать их по мере необходимости, для развивающихся стран они могут быть единственным выбором из-за малой мощности электрических сетей. Для Украины также может оказаться перспективным строительство энергоблоков SMR для замещения устаревших угольных энергоблоков и автономных источников производства электроэнергии и тепла для высокотемпературных технологических процессов.

\section{Теплофизические исследования для совершен- ствования легководных ядерных реакторов}

На данном этапе развития ядерной энергетики ставится задача повышения безопасности АЭС и улучшения их экономических и эксплуатационных характеристик путем сочетания свойств самозащищенности и пассивных систем безопасности. Разработка и обоснование таких систем требуют проведения теплофизических исследований работы ЯЭУ в стационарных и нестационарных режимах, включая экспериментальные исследования, разработку и совершенствование методов численного моделирования [33-36]. Разработка ядерных энергетических установок IУ 
поколения с высоким уровнем безопасности требует проведения фундаментальных исследований в области теплофизики. Основным направлением научных исследований в области гидродинамики и теплообмена для легководных ядерных реакторов остаются исследования кризиса теплообмена для новых вариантов геометрии тепловыделяющих сборок, нестационарных и аварийных режимов.

Надежно рассчитать гидродинамические процессы можно для однофазного течения теплоносителя. Для двухфазных сред возникают большие трудности с выбором модели турбулентности при расчетах локальных характеристик потока, замыкающих уравнений при расчетах осредненных параметров в нестационарных процессах. До сих пор нет надежных карт режимов двухфазных потоков для разного давления. Карты режимов течения, используемые в компьютерных кодах RELAP, CATHARE, KOРCAР и др., верифицированы экспериментальными данными для газожидкостных и пароводяных потоков низкого давления [36].

Для проектирования наиболее теплонапряженных областей активной зоны реакторов, парогенераторов и теплобменников необходимо знать не только средние значения характеристик теплоносителя, но и их распределение по сечению, а также учитывать возможные отклонения действительных значений характеристик от расчетных. Для этого нужны экспериментальные исследования с использованием новейшей измерительной техники.

При расчетах теплогидравлических характеристик тепловыделяющих сборок требуется учитывать массообмен между ячейками, прогибы и смещения тепловыделяющих элементов, влияние дистанционирующих решеток, неравномерность распределения теплоносителя по ячейкам. В Институте технической теплофизики существует действующий теплогидравлический стенд высоких параметров для исследования теплофизических процессов в элементах активных зон водоохлаждаемых ядерных реакторов (трубах, кольцевых каналах, пучках стержней). Стенд дает возможность проводить экспериментальные исследования кризиса теплообмена в каналах сложной геометрии в стационарных и не- стационарных режимах в диапазоне изменения массовой скорости от 120 до 3500 кг $/ \mathrm{M}^{2} \mathrm{c}$, давления до $18 \mathrm{MПа,} \mathrm{плотности} \mathrm{теплового} \mathrm{потока} \mathrm{до}$ 2,6 МВт/м². На основании экспериментальных данных, полученных в разное время, создана База данных по кризису теплообмена в трубах, кольцевых каналах, пучках стержней. В настоящее время проводятся фундаментальные исследования границ области режимов кипения в парогенерирующих каналах в широком диапазоне изменения давления [37,38], влияния смены режимов течения теплоносителя на энтальпию поперечных потоков между ячейками пучка стержней [39]. Полученные результаты экспериментальных исследований позволяют верифицировать математические модели и компьютерные программы, используемые для обоснования безопасности АЭС. Так экспериментальные данные по перемешиванию теплоносителя в ячейках тепловыделяющих сборок позволяют получить замыкающие уравнения для коэффициентов межканального тепло- и массопереноса, которые используются в поканальных (ячейковых) теплогидравлических кодах, а также при трехмерном моделировании активной зоны реакторов. Наличие базы данных по кризису теплообмена позволило верифицировать математические модели для исследования нестационарных и аварийных режимов в теплообменном оборудовании АЭС [40-42].

На основе разработанной трехмерной математической модели двухфазного потока и нестационарной RNG k-є модели турбулентности проведено численное исследование нестационарных теплофизических процессов в обогреваемых каналах при равномерной и неравномерной тепловой нагрузке $[15,43,44]$.

\section{Теплоперенос в наножидкостях при кипении}

Особый интерес в теплофизике вызывают наножидкости - суспензии (коллоиды) наночастиц в базовой жидкости. Благодаря высокой эффективности отвода теплоты пузырьковое кипение наножидкости является предпочтительным во многих промышленных процессах. Однако отсутствие понимания механизмов, ответственных за кардинальные изменения теплопередачи в жидкостях с добавлением наночастиц, и отсутствие моделей прогнозирования этих изменений 
препятствуют их широкому использованию.

По некоторым экспериментальным данным теплоперенос в наножидкостях увеличивается на $100 \ldots 120 \%$ по сравнению с исходной жидкостью [45]. Подобное явление нельзя описать моделями эффективной среды. Для объяснения основных эффектов аномального теплопереноса в наножидкостях использовались модели, учитывающие микроконвекцию, броуновское движение, наличие твердой пленки между жидкостью и наночастицами, различные модели кластеризации, модели внутренней радиационной передачи теплоты и т.П. Считается установленным, что при объемной доле наночастиц $0,1 \ldots 3,0$ \% эффективное увеличение теплопереноса составляет $3 \ldots 10 \%$ [45]. При кипении наножидкости на обогреваемой поверхности наблюдается как увеличение, так и уменьшение коэффициента теплоотдачи, при этом критический тепловой поток в наножидкости может быть больше, чем в чистой жидкости [46-49]. В работе [47] были исследованы особенности теплоотдачи при кипении наножидкости $\mathrm{Al}_{2} \mathrm{O}_{3}$ - вода на вертикальной и горизонтальной обогреваемой поверхности. Коэффициент теплоотдачи при кипении наножидкости был меньше, чем в чистой жидкости и уменьшался с увеличением концентрации наночастиц. В результате осаждения наночастиц на обогреваемой поверхности наблюдалось менее активное зарождение пузырьков. С другой стороны критический тепловой поток в наножидкости увеличился на $32 \%$ для горизонтальной и на $13 \%$ для вертикальной обогреваемой поверхности.

В Массачусетском технологическом институте проводятся исследования по использованию наножидкостей для систем безопасности легководных ядерных реакторов, в частности для залива активной зоны при тяжелой аварии с потерей теплоносителя. Использование наножидкости для охлаждения горячей активной зоны может существенно увеличить скорость ее охлаждения. Были проведены экспериментальные исследования с целью изучения механизмов влияния наночастиц на процессы закалки металлических сфер в наножидкостях [50]. Авторы приходят к выводу, что увеличение шероховатости поверхности и смачиваемости вследствие осаждения нано- частиц может быть ответственно за ускорение смены режима кипения от пленочного к пузырьковому и перехода к конвективному теплообмену.

Вопросы использования наножидкостей в качестве теплоносителя первого контура водоохладжаемых реакторов исследуются как с точки зрения теплофизики, так и нейтронной физики [51]. Так, при очень низкой концентрации наночастиц их влияние на свойства теплоносителя минимально. Однако с повышением концентрации наночастиц происходит уменьшение эффективного коэффициента размножения $K_{\text {eff }}$ и повышение отложений на стенках тепловыделяющих элементов. В диапазоне объемной доли наночастиц от 0,0001 до 0,1 наблюдается увеличение критического теплового потока и минимальное изменение коэффициента теплоотдачи пузырькового кипения. Результаты исследования изменения $K_{\text {eff }}$ показали его уменьшение с увеличением объемной доли наночастиц, причем этот эффект для различных типов наночастиц различен. Для нормальной работы реактора с небольшим изменением $K_{\text {eff }}$ наилучшей будет наножидкость $\mathrm{Al}_{2} \mathrm{O}_{3}$ - вода с объемной долей наночастиц 0,001 . Наножидкости $\mathrm{CuO}$ - вода в наибольше степени способствуют снижению $K_{\text {eff }}$. Отложения наночастиц на поверхности элементов активной зоны могут повысить безопасности ядерного реактора, но ценой снижения мощности.

Для выбора наиболее подходящих наножидкостей в качестве теплоносителя необходимы систематические исследования процессов тепломассопереноса в двухфазных потоках. В работах [52-54] представлены результаты теоретических исследований влияния наночастиц на процессы тепломассопереноса в наножидкостях при кипении и конденсации.

\section{Заключение}

Целью разработки новых ядерных технологий является решение проблем воспроизводства топлива, безопасности АЭС и обращения с радиоактивными отходами.

Внутренне присущая (естественная) безопасность открывает путь новой ядерной технологии, гармонически сочетающей качества безопасности, бридинга и экономичности.

Внедрение многокомпонентной структуры 
ядерной энергетики в будущем позволит разным типам реакторов наилучшим образом удовлетворять требования по сферам их применения (электричество, теплота, производство водорода, транспорт, наработка изотопов), уровню мощности (большие, средние и малые энергоисточники), спектру нейтронов (быстрые, тепловые, специальные реакторы-дожигатели долгоживущих радиоактивных отходов).

\section{ЛИТЕРАТУРА}

1. Осима К, Окубо О. Состояние и перспективы развития АЭС// Атомная техника за рубежом. 2010. - №12. - C.20 - 24.

2. Pioro I., Kirillov P. Current status of electricity generation at nuclear power plants/ http://www. formatex.info/energymaterialbook/book/. - 2013. P.806 - 817 .

3. Тюнин И.Б. Эволюционные и инновационные ядерные реакторы для ближайшей и отдаленной перспективы. Часть 1// Атомная техника за рубежом. - 2005. - №1. - С.3-10.

4. Ковецкий В.М., Ковецкая М.М. Направления развития технологий ядерных энергетических установок. Легководные реакторы// Проблеми загальної енергетики. - 2006. - №13. - С.29 - 37.

5. Ковецкий В.М., Ковецкая М.М. Направления развития технологий ядерных энергетических установок. Часть 2. Тяжеловодные, жидкометаллические и газовые реакторы// Проблеми загальної енергетики. - 2006. - №14. - С.14 -19.

6. Белозоров Д.П., Давыдов Л.Н. Современные проблемы ядерной энергетики: усовершенствованные реакторы Ш и Ш+ поколений//Вісник Харківського університету. - 2007. - №777. C. $3-32$.

7. Алексеев П.Н., Гагаринский А.Ю., Пономарев-Степной Н.Н., Сидоренко В.А. Требования к атомным станциям XX1 в.//Атомная энергия. 2000. - т.88, №1. - С.3 - 14.

8. Патон Б.Е., Неклюдов И.М., Красноруикий В.С. Будущее атомной энергетики определяет задачи ядерного топливного цикла Украины/ ISSN 15626016 BAHT. - 2013. - №5(87).

9. Pioro I., Kirillov P. Generation IY Nuclear Reactors as a Basis for Future Electricity Production in the World / http://www.formatex.info/ energymaterialbook/book/. - 2013. - p.818-830.
10. Тюнин И.Б. Эволюционные и инновационные ядерные реакторы для ближайшей и отдаленной перспективы. Часть 2// Атомная техника за рубежом. - 2005. - №2. - С.3-11.

11. Мачуи K. Тенденции разработок реакторов следующего поколения и реакторов нового типа в Японии// Атомная техника за рубежом . - 2004. №4. - C.24 - 37 .

12. Печера Ю. Международные инициативы в области разработок ЯЭУ и топливных циклов новых поколений // Атомная техника за рубежом. 2005. - №7. - c.21 - 26.

13. Колочко В., Авраменко А., Ковеикий В., Ковецкая M. Перспективы использования высокотемпературных газовых реакторов // Энергоатом Украины. - 2009. - №2. - С.16 - 19.

14. Бронников B.A. ЯЭУ на основе модуля газовая турбина-реактор с гелиевым (GT-MHR) теплоносителем // Атомная техника за рубежом. 2003. - №6. - C.20 - 22.

15. Авраменко А.А., Басок Б.И., Дмитренко Н.П., Ковецкая М.М., Тыринов А.И., Давыденко Б.В. Ренормализационно групповой анализ турбулентности. Киев. ТОВ «Видавничо- поліграфічний центр «ЕКСПРЕС» . - 2013. - 300с.

16. Дмитренко Н.П. Численное исследование теплообмена и гидродинамики в модельной активной зоне насыпного типа высокотемпературных газоохлаждаемых реакторов в условиях нестационарности // Промышленная теплотехника. - 2012. - т.34, №1. - С.48 - 52 .

17. Дмитренко Н.П. Исследование гидродинамических и теплофизических характеристик в тепловыделяющей сборке высокотемпературных газоохлаждаемых реакторов// Промышленная теплотехника. - 2009. - т.31, №4. - С.56 - 61 .

18. Ковецкая М.М., Дмитренко Н.П., Скиико А.И., Кондратьева Е.А. Процессы теплообмена при течении гелия и воды сверхкритического давления в тепловыделяющей сборке// Промышленная теплотехника. - 2014. - т.36, №2. - C. $46-53$.

19. Кириллов П.Л. Водоохлаждаемые реакторы на воде сверхкритических параметров// Теплоэнергетика. - 2008. - №5. - С. 2-5.

20. Cao L., Oka Y., Ishiwatari Y., Shang Z. Core Design and Subchannel Analysis of a Superfast Reactor// Journal of Nuclear Science and Technology. 
-2008. - v.45, № 2. - P.138-148.

21. Семечков Ю.М., Духовенский А.С., Алексеев П.Н. u др. Проблемы и перспективы реакторов нового поколения со сверхкритическим давлением// Теплоэнергетика.- 2008. - №5. - С.6-11.

22. Глебов А.П., Клушин А.В. Реактор с быстрорезонансным спектром нейтронов, охлаждаемый водой сверхкритического давления при двухходовой схеме движения теплоносителя// Атомная энергия. - 2006. - т.100, №5. - С. 349-355.

23. Драгунов Ю.Г., Рыжков С.Б., Никитенко М.П. и др. Водоохлаждаемые реакторы со сверхкритическими параметрами (ВВЭР-СКД) - перспективные реакторы 4-го поколения/ Труды конференции «Обеспечение безопасности АЭС с ВВЭР» Россия, Подольск. - 2007.

24. Смирнов В.П., Папандин М.В., Лонинов А.Я., Ванюкова Г.В., Афонин С.Ю. Применение CFD кода к расчету теплообмена в реакторе со сверхкритическими параметрами.// Атомная энергия. - 2011. -т.111, № 4.- С.196-201.

25. Блинков В.Н., Габараев Б.А., Мелихов О.И., Соловьев С.Л. Нерешенные проблемы тепло- и массообмена водоохлаждаемых реакторных установок со сверхкритическими параметрами теплоносителя. - М: ФГУП НИКИЭТ. - 2008. $85 \mathrm{c}$.

26. Авраменко А.А., Кондратьева Е.А., Ковеикая М.М., Тьринов А.И. Гидродинамика и теплообмен потока воды с сверхкритическими параметрами в вертикальной сборке тепловыделяющих элементов// Инженерно-физический журнал. - 2013. - т.86, №4. - С. 760-767.

27. Петрушин В.В., Гуреева Л.В., Фадеев Ю.П., Шмелев И.В., Лепехин А.Н., Удалищев С.В. Перспективы развития атомных станций с реакторами малой и средней мощности/ Сб. Атомные станции малой мощности т.2/ под ред. А.А. Саркисова/. - 2012. - С.36-49.

28. Чон-Кюн Парк. Атомные станции с передовыми реакторами малой и средней мощности потенциальный вариант энергоснабжения для выполнения конкретных функций: Вступительное слово от МАГАТЭ/ Сб. Атомные станции малой мощности т.2/ под ред. А.А. Саркисова/. 2012. - C.10-13.

29. Kidd S. SMRs - what are their prospects? // Nuclear Engineering International. - 2010. - v.66,
№ 677 . $-\mathrm{P} .12-13$.

30. Исаев А. Перспективы применения реакторов малой мощности с большой длительностью кампании// Атомная техника за рубежом. -2007. №6. - C.11-18.

31. Ковеикая М.М., Лаврик В.М., Ковеикий В.М. Проблемы и перспективы использования малых и средних ядерных реакторов// Проблеми загальної енергетики. - 2003. - №9. - С.32-36.

32. Fetterman R., Smith M., Harkntss A., Taylor $C$. Westinghouse SMR// Nuclear Engineering International. - 2012. - № 692. - P.16-19.

33. Ефанов А.Д., Калякин С.Г., Сорокин А.П. Теплофизические исследования в обоснование проектов ядерных реакторов нового поколения// Атомная энергия. - 2012. - т.112, №1. - С.12-18.

34. Кириллов П.Л. Основные направления научных исследований в области теплогидравлики атомных энергетических установок (Часть1)// Теплоэнергетика. - 2005. - №3. - С.15-19.

35. Кириллов П.Л. Основные направления научных исследований в области теплогидравлики атомных энергетических установок (Часть2)// Теплоэнергетика. - 2005. - №5. - С.47-52.

36. Гулевич А.В., Ефанов А.Д., Кириллов П.Л., Козлов Ф.А. Основные вопросы теплофизики ЯЭУ// Атомная энергия. - 2004. - т.96, №5. C.380-387.

37. Антипов В.Г. Экспериментальное определение границ области неравновесного кипения в парогенерирующем канале// Промышленная теплотехника. - 2011. - т.33, №6 . - С.25-31.

38. Антипов В.Г. Теплообмен в области неравновесного кипения воды в вертикальной трубе// Промышленная теплотехника. - 2015. - т.39, №3.C.16-2.3

39. Авраменко А.А., Кондратьева Е.А., Ковеикая М.М., Тыринов А.И. Влияние режимных параметров на энтальпию поперечных потоков между ячейками пучка стержней// Промышленная теплотехника. - 2015. - т.37, №3. - С.16-23.

40. Ковецкая М.М., Колесниченко Ю.М., Богорош A.T. Особенности нестационарного кризиса теплообмена в вертикальных парогенерирующих каналах// Промышленная теплотехника. - 2007. т.29, №1. - С.43-48.

41. Ковецкая М.М., Домашев В.Е., Ковецкая Ю.Ю. Исследование кризиса теплообмена в парогене- 
рирующем канале при набросе мощности// Промышленная теплотехника. - 2012. - т.34, №6. C.53-57.

42. Dolinsky A.A., Kovetskaya M.M., Skitsko A.I., Avramtnko A.A., Basok B.I. Nonstationary Heat Transfer Crisis in Annular Dispersed Flows// Journal of Engineering Thermo physics. - 2008. - v.17, №2 . P.126-129.

43. Скиџко А.И., Ковецкая М.М., Тыринов А.И. Численное исследование тепломассопереноса в вертикальном парогенерирующем канале под влиянием возмущающих факторов// Промышленная теплотехника. - 2013. - т.35, №6. - С.9-15. 44. Ковеикая М.М., Кондратьева Е.А., Скицко А.И. Влияние неравномерности тепловой нагрузки на локальные характеристики потока при течении воды в парогенерирующих каналах и тепловыделяющих сборках// Промышленная теплотехника. - 2014. - т.36, №3. - С.38-44.

45. Дмитриев А.С. Теплофизические проблемы наноэнергетики. Часть 2// Теплоэнергетика. 2011. - №4. - C.29-36.

46. Ramesh G., Prabhu N.K. Review of thermophysical properties, wetting and heat transfer characteristics of nanofluids and their applicability in industrial quench heat treatment//Nanoscale Research Letters. - 2011. - 6:334. - P.1-15.

47. Bang I.C., Chang S.H. Boiling Heat transfer performance and phenomena of $\mathrm{Al}_{2} \mathrm{O}_{3}$ - water nanofluids from a plain surface in a pool// International Journal of Heat and Mass Transfer. -
2005. - 48. - P.2407-2419.

48. Wang X.Q, Mujumdar A.S. Heat transfer characteristics of nanofluids: a review// International Journal of Thermal Sciences. - 2007. - 46. - P.1-19. 49. Das S.K., Choi S.U.S., Patel H.E. Heat Transfer in Nanofluids - A Review//Heat Transfer Engineering. - 2006. - 27(10). - P.3-19.

50. Kim H., DeWitt G., McKrell T., Buongiorno J., $H u L . W$. On the quenching of steel and zircaloy spheres in water- based nanofluids with alumina, silica and diamond nanoparticles// International Journal of Multiphase Flow. - 2009. - 35 . - P.427438.

51. Hadad K., Hajizadeh A., Jafarpour K., Hanapol B.D. Neutronic study of nanofluids application to VVER1000// Annals of Nuclear Energy. - 2010. - v.37, №11. - P.1447-1455.

52. Avramenko A.A., Tyrinov A.I. Heat transfer at film condensation of moving vapor with nanoparticles over a flat surface// International Journal of Heat and Mass Transfer. - 2015. - 82. - P.316-324. Impact Factor:2.809.

53. Avramenko A.A., Tyrinov A.I. Heat transfer in stable film boiling of a nanofluid over a vertical surface// International Journal of Thermal Sciences. - 2015. - 92. - P.106-118. - Impact Factor: 3.156.

54. Avramenko A.A., Tyrinov A.I. Thermocapillary instability in an evaporating two-dimensional thin layer film// International Journal of Heat and Mass Transfer. - 2015. - 91. - P.77-88. - Impact Factor:2.809. 


\section{PERSPECTIVE RESEARCHES OF INNOVATIVE TECHNOLOGIES OF NUCLEAR POWER PLANTS}

\section{A.A. Avramenko, M.M. Kovetskaya, A.V. Kravchuk, Yu.Yu. Kovetskaya}

Institute of Engineering Thermophysics of the National Academy of Sciences of Ukraine, 03057, Kiev, vul. Zhelyabova 2a, Ukraine.

A review of perspective nuclear power technologies is presented for solving the problems of improving the safety and economic efficiency. The development of new nuclear technologies aimed at solving problems of reproduction of fuel, nuclear safety atomic electrical station and radioactive waste management. In future introduction of a multi-component structure of nuclear power will allow creating different types of reactors with best properties. A role of thermal physical investigation for $i$ mproving light-water reactors is shown.

Key words: new generation of nuclear reactors, helium coolant, water supercritical pressure, thermophysical studies.

References 54, tabl. 5.

1. Oshima K., Okubo O. The condition and prospects of development of nuclear power plants // Atomic energy technology overseas. - 2010. - №12. - P.20 24. (Rus.)

2. Pioro I., Kirillov P. Current status of electricity generation at nuclear power plants/ http://www. formatex.info/energymaterialbook/book/. - 2013. P.806- 817 .

3. Tyunin I.B. The evolutionary and innovative nuclear reactors for the near and distant future. Part $1 / /$ Atomic energy technology overseas. - 2005. №1. - C. 3 - 10. (Rus.)

4. Kovetskiy V.M., Kovetskaya M.M. Directions of development of nuclear power plant technology. Light water reactors // Problems of general power industry. - 2006. - №13. - C.29 - 37. (Rus.)

5. Kovetskiy V.M., Kovetskaya M.M. Directions of development of nuclear power plant technology. Part 11. Heavy water, liquid metal reactors and gas // Problems of general power industry. - 2006. - №14.
- P.14-19 (Rus.)

6. Belozorov D.P., Davydov L.N. Current problems of nuclear power: III and III+ generations advanced reactors // Kharkiv University Herald. - 2007. №777. - P. 3 - 32. (Rus.)

7. Alekseev P.N., Haharynskyy A.Yu., PonomarevStepnoy N.N., Sidorenko V.A. Demands for nuclear power plants in XXI c. // Nuclear energy . - 2000. V.88, №1 - P. 3 - 14. (Rus.)

8. Paton B.Ye., Neklyudov I.M., Krasnorutskyy V.S. The future of nuclear power determines tasks of Ukraine's nuclear fuel cycle // ISSN 1562-6016 VANT. - 2013. - №5(87). - P. 3 - 10. (Rus.)

9. Pioro I., Kirillov P. Generation IY Nuclear Reactors as a Basis for Future Electricity Production in the World / http://www.formatex.info/ energymaterialbook/book/ - 2013 - P. $818-830$.

10. Tyunin I.B. The evolutionary and innovative nuclear reactors for the near and distant future. Part 2 // Atomic energy technology overseas. - 2005. №2. - C.3 - 11. (Rus.)

11. Matsui K. Trends in development of the next generation of reactors and new reactors in Japan // Atomic energy technology overseas. - 2004. - №4. P. 24 - 37. (Rus.)

12. Pechera Yu. International initiatives in the development of nuclear power and fuel cycle of new generations // Atomic energy technology overseas. 2005. - №7. - P. 21 - 26. (Rus.)

13. Kolochko V., Avramenko A., Kovetskiy V., Kovetskaya $M$. Prospects for the use of hightemperature gas reactors // Energoatom Ukraine. 2009. - №2. - P. 16 - 19. (Rus.)

14. Bronnikov V.A. NPP on the basis of the gas turbine unit with helium reactor (GT-MHR) coolant // Atomic energy technology overseas. - 2003. №6. - P.20 - 22. (Rus.)

15. Avramenko A.A., Basok B.I., Dmitrenko N.P., Kovetskaya M.M., Tyrinov A.I., DavydenkoB.V. Renormalization group analysis of turbulence. - K.: Expres. - 2013. - 299p. (Rus.)

16. Dmitrenko N.P. Numerical investigation of heat transfer and hydrodynamics in the model core bulktype high-temperature gas-cooled reactors under unsteadiness //// Industrial heat engineering. - 2012. V.34, №1. - P.48 - 52. (Rus.)

17. Dmitrenko N.P. The study of hydrodynamic and thermal characteristics in the fuel assembly of high- 
temperature gas-cooled reactors // Industrial heat engineering. - 2009. - V.31, №4. - P.56 - 61. (Rus.)

18. Kovetskaya M.M., Dmitrenko N.P., Skitsko A.I., Kondratieva E.A. Heat transfer processes in the flow of helium and the supercritical water in the fuel assembly // Industrial heat engineering.- 2014. V.36, №2. - P.46 - 53. (Rus.)

19. Kirillov P.L. Water-cooled reactors, supercritical water // Teploenergetika. - 2008. - №5. - P.2 - 5. (Rus.)

20. Cao L., Oka Y., Ishiwatari Y., Shang Z. Core Design and Subchannel Analysis of a Superfast Reactor//JournalofNuclearScienceand Technology.2008. -V.45, № 2. - P.138-148.

21. Semechkov Yu.M., Duhovensky A.S., Alekseev P.N. Problems and prospects of the new generation of reactors with supercritical pressure // Teploenergetika. - 2008. - №5. - P.6 - 11. (Rus.)

22. Glebov A.P., Klushin A.V. The reactor with the fast-neutron resonance spectrum cooled supercritical water at a two-way movement of the coolant circuit // Nuclear energy. - 2006. - V.100, №5. - C.349 355. (Rus.)

23. Dragunov Yu.G., Ryzhov S.B., Nikitenko M.P. Water-cooled reactors with supercritical parameters (WVER-SKD) - prospective reactors of the 4th generation / Conference proceedings "Ensuring the safety of nuclear power plants with VVER" Russian, Podolsk. - 2007. (Rus.)

24. Smirnov V.P., Papandin M.V., Loninov A.Y., Vanyukova G.V., Afonin S.Yu. Application of CFD code to the calculation of heat transfer in the reactor with supercritical parameters / Nuclear energy. 2011. - V.111, № 4.- C.196 - 201. (Rus.)

25. Blinkov V.N., Gabaraev B.A., Melikhov O.I., Soloviev S.L. Unsolved problems of heat and mass transfer of water-cooled reactor plants with supercritical coolant parameters. - M: FGUP NIKIET. - 2008. - 85p. (Rus.)

26. Avramenko A.A., Kondratieva E.A., Kovetskaya M.M., Tyrinov A.I. Hydrodynamics and heat flow with supercritical parameters in the vertical assembly of fuel elements // Journal of Engineering Physics. - 2013. - V.86, №4. - C.760 - 767. (Rus.)

27. Petrushin V.V., Gureeva L.V., Fadeev Yu.P., Shmelev I.V., Lepekhin A.N., Udalischev S.V. Prospects for the development of nuclear power plants with reactors of low and medium power /
Nuclear power station small / Ed. Sarkisov /. - 2012. - V.2 - P.36 - 49. (Rus.)

28. Jong-Kyun Park. Nuclear power plants with advanced reactors of low and medium power - energy potential option for a specific function: Opening remarks by MAGATE / Nuclear power station small / Ed. Sarkisov /. - 2012. - V.2 - P.10 -13. (Rus.)

29. Kidd S. SMRs - what are their prospects? // Nuclear Engineering International. - 2010. -V.66, № 677 . - P.12 - 13 .

30. Isayev A. Prospects for the use of low-power reactors with a long duration of the campaign // Atomic energy technology overseas. - 2007. - №6. C.11 - 18. (Rus.)

31. Kovetskaya M.M., Lavrik V.M., Kovetskiy V.M. Problems and prospects of small and medium-sized nuclear reactors // Total problems of zagalnoï power industry. - 2003. - №9. - C.32 - 36. (Rus.)

32. Fetterman $R$., Smith M., Harkntss A., Taylor $C$. Westinghouse SMR// Nuclear Engineering International. - 2012. - № 692. -P.16 - 19.

33. Efanov A.D., Kalyakin S.G., Sorokin A.P. Thermal studies in support of projects of nuclear reactors of new generation// Nuclear energy. - 2012.V.112, №1. - P.12 - 18. (Rus.)

34. Kirillov P.L. The main directions of research in the field of thermal hydraulics of nuclear power plants Research (Part 1) // Teploenergetika. - 2005. №3. - C.15 - 19. (Rus.)

35. Kirillov P.L. The main directions of research in the field of thermal hydraulics of nuclear power plants Research (Part 2) // Teploenergetika. - 2005. №5. - C.47 - 52. (Rus.)

36. Gulevich A.V., Efanov A.D., Kirillov P.L., Kozlov F.A. The main issues of Thermal Physics of nuclear power// Nuclear energy. - 2004. -V.96, №5. C. $380-387$. (Rus.)

37. Antipov V.G. Experimental determination of the boundaries of the field of nonequilibrium boiling in the steam generating channel // Industrial heat engineering. - 2011. -V.33, №6 . - C. 25-31. (Rus.)

38. Antipov V.G. Heat transfer in a nonequilibrium boiling water in a vertical tube // Industrial heat engineering. - 2015. -V.39, №3. - C. 16-23. (Rus.) 39. Avramenko A.A., Kondratieva E.A., Kovetskaya M.M., Tyrinov A.I. Influence of regime parameters on the enthalpy of the cross flows between cell bars beam // Industrial heat engi- 
neering. - 2015. - V.37, №3. - C. 16 -23. (Rus.)

40. Kovetskaya M.M., Kolesnichenko Yu.M., Bogorosh A.T. Features of unsteady heat transfer crisis in the vertical steam-generating channels // Industrial heat engineering. - 2007. -V.29, №1. C. 43 - 48. (Rus.)

41. Kovetskaya M.M., Domashev V.E., Kovetskaya $Y u$., Yu. The study of heat transfer crisis in the steam generating channel at pounce power // Industrial heat engineering. - 2012. -V.34, №6. - C.53 - 57. (Rus.)

42. Dolinsky A.A., Kovetskaya M.M., Skitsko A.I., Avramtnko A.A., Basok B.I. Nonstationary Heat Transfer Crisis in Annular Dispersed Flows // Journal of Engineering Thermo physics. -2008. -V.17, №2. P.126- 129 .

43. Skitsko A.I., Kovetskaya M.M., Tyrinov A.I. Numerical study of heat and mass transfer in a vertical steam generating channel under the influence of disturbing factors // Industrial heat engineering. 2013. -V.35, №6. - C.9 - 15. (Rus.)

44. Kovetskaya M.M., Kondratieva E.A., Skitsko A.I. Effect of uneven heat load on the local characteristics of the flow during flow of water in the steam generating channel and the fuel assemblies // Industrial heat engineering. - 2014. -V.36, №3. - C.38 - 44. (Rus.) 45. Dmitriev A.S. The thermophysical problems nanoenergy. Part 2 // Teploenergetika. - 2011. - №4. - C.29 - 36. (Rus.)

46. Ramesh G., Prabhu N.K. Review of thermophysical properties, wetting and heat transfer characteristics of nanofluids and their applicability in industrial quench heat treatment // Nanoscale Research Letters. - 2011. - 6:334. - P.1-15.

47. Bang I.C., Chang S.H. Boiling Heat transfer performance and phenomena of A12O3 - water nanofluids from a plain surface in a pool // International Journal of Heat and Mass Transfer. -2005. - №48. - P. 2407 - 2419.

48. Wang X.Q, Mujumdar A.S. Heat transfer characteristics of nanofluids: a review // International Journal of Thermal Sciences. -2007. - №46. - P.119.

49. Das S.K., Choi S.U.S., Patel H.E. Heat Transfer in Nanofluids - A Review // Heat Transfer Engineering. -2006. -V.27, №10. - P.3-19.

50. Kim H., DeWitt G., McKrell T., Buongiorno $J$., Hu L.W. On the quenching of steel and zircaloy spheres in water- based nanofluids with alumina, silica and diamond nanoparticles // International Journal of Multiphase Flow. - 2009. - №35 . - P.427 $-438$.

51. Hadad K., Hajizadeh A., Jafarpour K., Hanapol B.D. Neutronic study of nanofluids application to VVER-1000 // Annals of Nuclear Energy. -2010. V.37, №11. -P.1447-1455.

52. Avramenko A.A., Tyrinov A.I. Heat transfer at film condensation of moving vapor with nanoparticles over a flat surface // International Journal of Heat and Mass Transfer. - 2015. - №.82. - P.316 - 324. Impact Factor: 2.809.

53. Avramenko A.A., Tyrinov A.I. Heat transfer in stable film boiling of a nanofluid over a vertical surface // International Journal of Thermal Sciences. - 2015. - №.92. - P.106 -118. - Impact Factor: 3.156.

54. Avramenko A.A., Tyrinov A.I. Thermocapillary instability in an evaporating two-dimensional thin layer film // International Journal of Heat and Mass Transfer. - 2015. - №.91. -P.77-88. - Impact Factor: 2.809 . 